\title{
Penetrating trauma and invasive management of thorax trauma
}

\author{
L. P. H. Leenen ${ }^{1}$
}

Published online: 19 November 2018

c) Springer-Verlag GmbH Germany, part of Springer Nature 2018

Diagnostic and treatment modalities in trauma, as well as logistics and pre-hospital trauma care, are a fast-developing and widely discussed area of medicine. Although penetrating trauma is far less common in Europe than in many other parts of the world, there is a sense that it is on the rise in Europe as well. In the United States, the number of casualties from gunshot wounds is higher than from motor vehicle accidents-in short, scary statistics!

Nevertheless, penetrating trauma warrants further attention from the trauma surgeon in the widest sense. In this issue of the European Journal of Trauma and Emergency Surgery, we focus on penetrating trauma and invasive management of thoracic trauma.

Zanobetti et al. [1] evaluate the use of focused assessment sonography in trauma (FAST) for torso trauma. The increasingly widespread use of CT as the prime diagnostic modality has given rise to concerns regarding the radiation burden in trauma patients and the indiscriminate use of CT. Other modalities such as ultrasound have been widely discussed; however, they too have their downsides, such as operator dependence. Various reports on accuracy and negative predictive value show quite different results. Those reported by Zanobetti et al. are promising, and challenge the indiscriminate use of CT. They advise the use of ultrasound as a triage tool before initiating CT scanning in the trauma patient with suspicion of torso trauma.

One heavily discussed subject is resuscitative thoracotomy. Challenged not only at the moment by the new kid on the block, resuscitative endovascular balloon occlusion of the aorta (REBOA), its use for indications other than penetrating chest trauma is also under scrutiny. Its pre-hospital use is especially questionable. Nevins et al. [2] performed a MEDLINE and PubMed search on the subject and report fairly disappointing results from the literature. They

\section{P. H. Leenen}

1.p.h.leenen@umcutrecht.nl

1 Department of Surgery, University Medical Center Utrecht, Utrecht, Netherlands scrutinize current practices in their contribution to this journal. Readers must draw their own conclusions as to whether this procedure is appropriate for their individual practice environments based on this result; however, one has to consider cost and training efforts, as well as who should perform these procedures in the field.

Do you perhaps treat the tube drainage of the chest as you learned it during residency, without asking yourself whether there are choices in using suction drainage or simply using a water seal? Feenstra et al. [3] performed a systematic review and meta-analysis to answer this question and have come up with remarkable results, as discussed in this very readable article.

Another question which seems to have no clear answer, however, involves patient discharge. In current crowded emergency departments, early discharge of the patient home is a welcome addition for patients with penetrating chest injury. Seidzadeh Gooklan et al. [4] put up a fairly straightforward comparison after 1 and $3 \mathrm{~h}$ of observation, revealing that no further complications occurred after the initial $\mathrm{X}$-ray of the chest. They show that early observation without further complications warrants early discharge. However, the authors confine their results to a fairly limited group, as several prerequisites are made for their conclusions, which leaves the question as to how many patients this will apply to on an average Saturday evening.

Asensio et al. [5] publish a review of a very special group of patients, those with penetrating cardiac injury, a largely deadly injury. From a detailed (American) National Trauma Data Bank (NTDB) evaluation, they extract prognostic elements to build a predictive model for the outcome of these patients. Among the 1,310,720 patients included in the 2016 database, they identify $0.16 \%$ with penetrating injuries of the heart. From this they present a predictive model that can provide direction for further guidelines in treating these patients. 


\section{Compliance with ethical standards}

Conflict of interest The author has no conflicts of interest on the subjects presented here.

\section{References}

1. Zanobetti M, Coppa A, Nazerian P, et al. Chest abdominal-focused assessment sonography for trauma during the primary survey in the emergency department: the CA-FAST protocol. Eur J Trauma Emerg Surg. 2015. https://doi.org/10.1007/s00068-015-0620-y.

2. Nevins EJ, Moori PL, Smith-Williams J, et al. Should pre-hospital resuscitative thoracotomy be reserved only for penetrating chest trauma? Eur J Trauma Emerg Surg. 2018. https://doi.org/10.1007/ s00068-018-0937-4.

3. Feenstra TM, Dickhoff C, Deunk J. Systematic review and metaanalysis of tube thoracostomy following traumatic chest injury; suction versus water seal. Eur J Trauma Emerg Surg. 2018. https ://doi.org/10.1007/s00068-018-0942-7.

4. Seidzadeh Gooklan L, Yari A, Mayel M, et al. Observation period for asymptomatic penetrating chest trauma: 1 or $3 \mathrm{~h}$ ? Eur J Trauma Emerg Surg. 2016. https://doi.org/10.1007/s00068-015-0623-8.

5. Asensio JA, Ogun OA, Petrone $\mathrm{P}$, et al. Penetrating cardiac injuries: predictive model for outcomes based on 2016 patients from the National Trauma Data Bank. Eur J Trauma Emerg Surg. 2017. https://doi.org/10.1007/s00068-017-0806-6. 